\title{
The Plasmacytoid Dendritic Cell CD123+ Compartment in Acute Leukemia with or without RUNX1 Mutation: High Inter-Patient Variability Disclosed by Immunophenotypic Unsupervised Analysis and Clustering
}

\author{
Anna Porwit ${ }^{1, *}$ and Marie C. Béné ${ }^{2}$ (D) \\ 1 Department of Clinical Sciences, Division of Oncology and Pathology, Faculty of Medicine, Lund University, \\ 22185 Lund, Sweden \\ 2 Hematology Biology, Nantes University Hospital and CRCINA, 44000 Nantes, France; \\ mariecbene@gmail.com \\ * Correspondence: anna.porwit@med.lu.se
}

check for

updates

Citation: Porwit, A.; Béné, M.C. The Plasmacytoid Dendritic Cell CD123+ Compartment in Acute Leukemia with or without RUNX1 Mutation:

High Inter-Patient Variability

Disclosed by Immunophenotypic

Unsupervised Analysis and

Clustering. Hemato 2021, 2, 572-585.

https://doi.org/10.3390/

hemato2030036

Academic Editor: Roland B. Walter

Received: 30 June 2021

Accepted: 25 August 2021

Published: 1 September 2021

Publisher's Note: MDPI stays neutral with regard to jurisdictional claims in published maps and institutional affiliations.

Copyright: (c) 2021 by the authors. Licensee MDPI, Basel, Switzerland. This article is an open access article distributed under the terms and conditions of the Creative Commons Attribution (CC BY) license (https:/ / creativecommons.org/licenses/by/ $4.0 /)$.

\begin{abstract}
Plasmacytoid dendritic cells (PDC) constitute a small subset of normal bone marrow (BM) cells but have also been shown to be present, sometimes in large numbers, in several hematological malignancies such as acute myeloid leukemia with RUNX1 mutation, chronic myelomonocytic leukemia or, obviously, blastic plasmacytoid dendritic cell neoplasms. These cells have been reported to display somewhat variable immunophenotypic features in different conditions. However, little is known of their plasticity within individual patients. Using an unsupervised clustering tool (FlowSOM) to re-visit flow cytometry results of seven previously analyzed cases of hematological malignancies (6 acute myeloid leukemia and one chronic myelomonocytic leukemia) with a PDC contingent, we report here on the unexpectedly high variability of PDC subsets. Although five of the studied patients harbored a RUNX1 mutation, no consistent feature of PDCs could be disclosed as associated with this variant. Moreover, the one normal single-node small subset of PDC detected in the merged file of six normal BM could be retrieved in the remission BM samples of three successfully treated patients. This study highlights the capacity of unsupervised flow cytometry analysis to delineate cell subsets not detectable with classical supervised tools.
\end{abstract}

Keywords: flow cytometry; acute myeloid leukemia; unsupervised clustering; plasmacytoid dendritic cell; RUNX1

\section{Introduction}

Acquired mutations of the runt-related transcription factor 1 (RUNX1) gene are found in $5-15 \%$ of patients with de novo acute myeloid leukemia (AML) and myelodysplastic syndromes (MDS) or myelodysplastic/myeloproliferative neoplasms (MDS/MPN) such as chronic myelomonocytic leukemia (CMML) [1,2]. AML with RUNX1 mutation (RUNX1 ${ }^{\text {mut }}$ ) is a provisional entity in the WHO 2016 classification of hematopoietic neoplasms [3], to be considered after excluding other AML categories such as AML with recurrent genetic abnormalities, AML with MDS-related changes (AML-MRC) and therapy-related AML (t-AML). RUNX1 ${ }^{\text {mut }}$ AML is most often purely blastic by cytological assessment and myeloperoxidase-negative, corresponding to AML with minimal differentiation [2-4]. In some leukemias with RUNX1 ${ }^{\mathrm{mut}}$, mixed-phenotype acute leukemia (MPAL) characteristics can, however, be present [5], and RUNX1 ${ }^{\text {mut }}$ has also been associated with AML cases with a plasmacytoid dendritic cell (PDC) component [6,7]. However, RUNX1 ${ }^{\text {mut }}$ has only seldom been reported in blastic PDC neoplasms (BPDCN) [6,8], while clusters of mature PDCs have been described in bone marrow (BM) biopsies from patients with MPN or MDS/MPN [9]. An association of BPDCN with CMML has also been reported [10,11]. 
Finally, RUNX1 ${ }^{\text {mut }}$ has been found in blast crisis of chronic myeloid leukemia (CML) and RUNX1 ${ }^{\text {mut }}$ BP-CML patients showed a notable population of PDCs [12].

Conventional multiparameter flow cytometry (MFC) initial immunophenotypic definition of PDC was of cells negative for lineage markers (lin) coexpressing CD4 and CD56 [13,14]. Subsequently, these cells were recognized to express CD38, CD123, CD303 and HLA-DR, with a variable expression of CD33 and absence of CD64 [15-17]. Small fractions of normal/reactive PDCs positive for CD2, CD7 and/or CD56 have also been noted [18]. Dendritic cell progenitors in the human BM are extremely rare and their immunophenotype as well as stages of differentiation remain a matter of debate $[19,20]$. Blasts in BPDCNs with leukemic presentation show an immunophenotype similar to normal pCDs i.e., CD45dim/low SSC, CD4 $4^{+}$bright), $\mathrm{CD} 123^{+}$(bright), $\mathrm{CD} 56^{+}$(heterogenous), $\mathrm{CD}^{+} 3^{+}\left(\mathrm{dim}^{\prime}\right.$ heterogeneous), $\mathrm{CD}^{+} 6^{+}, \mathrm{CD} 38^{+}, \mathrm{HLA}^{-\mathrm{DR}^{+}}$and are negative forCD10, CD11b, CD13, CD14, CD15, CD16, CD19, CD34, CD64, CD65, CD235a, cyt.MPO, cyt.CD3, cyt.CD22, and nTdT [21].

The identification of PDC in MFC relies on sequential supervised gating strategies and monoparametric or biparametric histograms tracking cells with these suspected immunophenotypic features [19]. The recent development of tools for an unsupervised delineation of cell subsets within samples stained with targeted antibody panels allows for more accurate identification of discrete cell subsets in normal BM and for detailed studies of the heterogeneity of malignant proliferations [22,23]. FlowSOM is one of the new types of software, developed in the Bioconductor R system [24]. This software has been applied to the definition of normal BM and hematological malignancies with myeloid, lymphoid, and erythroid antibody panels [25-27]. Here we explored how FlowSOM could dissect PDC-like populations in seven patients with leukemic disorders involving these peculiar cells.

\section{Materials and Methods}

Bone marrow samples: Six normal bone marrow (NBM) samples were obtained from five healthy volunteers and one patient with idiopathic thrombocytopenia and reactive bone marrow. These six NBM samples were analyzed with AML MFC panels (Supplementary Table S1, [28]) at the Flow Cytometry Laboratory Lund's University Hospital Pathology Department, Sweden, as a part of a quality assurance study approved by the local ethics committee.

The same panel was applied for diagnostic purposes to BM samples from 7 AML patients with potential PDC involvement, tested between 2018 and 2021 according to routine procedures that included morphology, MFC, karyotype and molecular analyses. Patient characteristics are given in Table 1. The samples had been collected from patients hospitalized for an hematological malignancy who provided consent for diagnosis and management procedures, including flow cytometry. Only the listmode files stored in silico were used for this study and samples were de-identified, which waived the need for further consent. The clinical charts of the patients were not reviewed.

MFC methodology is given in Supplementary Methods and Supplementary Table S1. Karyotype analysis was performed by routine methods at the Department of Clinical Genetics, Lund University Hospital. Next-generation sequencing (NGS) was performed as a part of routine workup, using Illumina Trusight Myeloid sequencing panel (Illumina, San Diego, CA) according to manufacturer's recommendations at the Diagnostic Molecular Center, Lund University Hospital, Lund, Sweden. This commercialy available set of reagents targets exons from 15 genes and hotspots from 39 others in a comprehensive screening of 59 variants. Diagnostic criteria of the WHO 2016 classification were applied [3]. The samples were selected for detailed FlowSOM analysis based on the presence of RUNX1 ${ }^{\text {mut }}$ and/or increased numbers of $\mathrm{CD} 123^{+}$cells in routine MFC analysis. 
Table 1. Patient characteristics.

\begin{tabular}{|c|c|c|c|c|c|c|c|c|c|c|c|}
\hline Case \# & $\begin{array}{l}\text { Age } \\
\text { (Years) }\end{array}$ & Sex & $\mathrm{Hb} \mathrm{g} / \mathrm{L}$ & $\mathrm{WBC} \times 10^{9} / \mathrm{L}$ & Plt $\times 10^{9} / \mathrm{L}$ & $\begin{array}{l}\text { \% Blasts by } \\
\text { Cytology }\end{array}$ & $\begin{array}{c}\text { Diagnosis WHO } \\
2016\end{array}$ & Karyotype & $\begin{array}{l}\text { Molecular Data } \\
\text { (VAF) }^{\text {a }}\end{array}$ & $\% \mathrm{CD} 34^{\mathrm{b}}$ & $\% C D 123^{b}$ \\
\hline 1 & 73 & M & 59 & 35 & 12 & $88 \%$ & $\begin{array}{l}\text { RUNX1 } 1^{\mathrm{mut}} \\
\quad \mathrm{AML}\end{array}$ & $46, X Y$ & $\begin{array}{c}\text { RUNX1 46\%, IDH2 } \\
45 \%, \text { TET2 47\%, } \\
\text { SRSF2 50\% }\end{array}$ & $90 \%$ & $88 \%$ \\
\hline 2 & 75 & $\mathrm{~F}$ & 94 & 5.6 & 25 & $20 \%$ & $\begin{array}{c}\text { RUNX1 } 1^{\text {mut }} \\
\text { AML PDC } \\
\text { differentiated }\end{array}$ & NA * & $\begin{array}{c}\text { RUNX1 41\%, ASXL1 } \\
35 \%, \text { IDH2 48\%, NRAS } \\
45 \%, \text { SRSF2 } 45 \%\end{array}$ & $21 \%$ & $12 \%$ \\
\hline 3 & 29 & M & 114 & 2.5 & 149 & $31 \%$ & AML-MRC & $\begin{array}{l}\text { 46XY, del 7(q32), } \\
\text { ?inv.dup.21 } \\
\text { (q22;q11) } \\
\text { [19]/46,XY [9] }\end{array}$ & $\begin{array}{l}\text { RUNX1 53\%, } \\
\text { WT1 10\% }\end{array}$ & $30 \%$ & $4 \%$ \\
\hline 4 & 79 & M & 93 & 1.6 & 28 & $64 \%$ & $\mathrm{t}-\mathrm{AML}$ & $\begin{array}{l}47, \mathrm{XY},+8[14] / 48 \\
\mathrm{XY},+8,+8[11]\end{array}$ & $\begin{array}{c}\text { RUNX1 45\%, IDH2 } \\
\text { 41\%, SRSF2 44\%, } \\
\text { CUX1 5\% }\end{array}$ & $84 \%$ & $10 \%$ \\
\hline 5 & 71 & $\mathrm{~F}$ & 134 & 4.4 & 92 & $19 \%$ & $\begin{array}{l}\mathrm{CMML}^{* *} \text { and } \\
\text { BPDCN }\end{array}$ & $46, X X$ & $\begin{array}{c}\text { RUNX1 41\%, 2xTET2 } \\
\text { (50 and 46\%), PHF6 } \\
36 \%, \text { SRSF2 50\%, } \\
\text { NRAS } 10 \%,\end{array}$ & $9 \%$ & $3 \%$ \\
\hline 7 & 62 & M & 96 & 8.3 & 84 & $81 \%$ & $\begin{array}{c}\text { MPAL } \\
\text { (T/Myeloid) }\end{array}$ & $46, \mathrm{XY}, \operatorname{add}(14)(\mathrm{q} 32)$ & $\begin{array}{c}2 \times F L T 3(21 \% \text { and } 5 \%), \\
\text { DNMT3A }(48 \%), \\
\text { ASXL1 }(47 \%), J A R I D 2 \\
(15 \%), 2 \times B C L 11 B(9 \% \\
\text { and } 5 \%)\end{array}$ & $55 \%$ & $4 \%$ \\
\hline
\end{tabular}

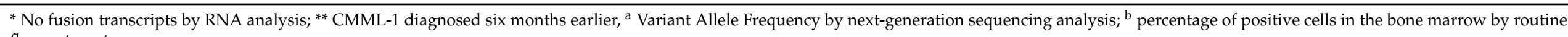
flow cytometry. 
FlowSOM analysis: For detailed analysis of PDC maturation, the raw (.fcs) files of panel AML 2 that included CD123 together with immature progenitor markers CD34 and CD117, as well as CD7, HLA-DR, CD38, CD33, CD45, and markers of monocyte maturation CD36 and CD64 were used (Supplementary Table S1). Analysis was performed on the Kaluza software (Beckman Coulter, Miami, FL, USA) equipped with FlowSOM plug-ins as previously described [25]. Briefly, all LMD files were checked for proper compensation and fluorescence was normalized by comparison to lymphocytes with a dedicated R script as reported [25]. The six normalized list-mode files of NBM samples were merged with the Kaluza merging tool. The resulting single merged NBM file was submitted to unsupervised analysis by FlowSOM with the set-seed option allowing the software to generate 24 minimal spanning trees (MST) of 100 nodes. Nodes with CD123 positivity were detected on the MST through back-gating and the major NBM subsets were determined by coloring a classical CD45/SSC dot plot as previously described [25]. The graphical MST providing the best grouping, yet clear separation of the nodes of interest was then chosen (Figure 1). Each node/group of nodes of interest was individually studied in Kaluza, recording the number of events, scatter properties and normalized mean fluorescence intensity (MFI) of each marker. The frequency of cells in each node/group of nodes was then calculated as a fraction of the whole sample. Based on the analysis of the NBM merged file, and cut-offs for normalized marker expression were established (Supplementary Table S2). The cut-off for positive CD123 normalized expression was a mean fluorescence intensity (MFI) of 2.0, while cells with a normalized expression $>3.0$ were considered as strongly CD123-positive.
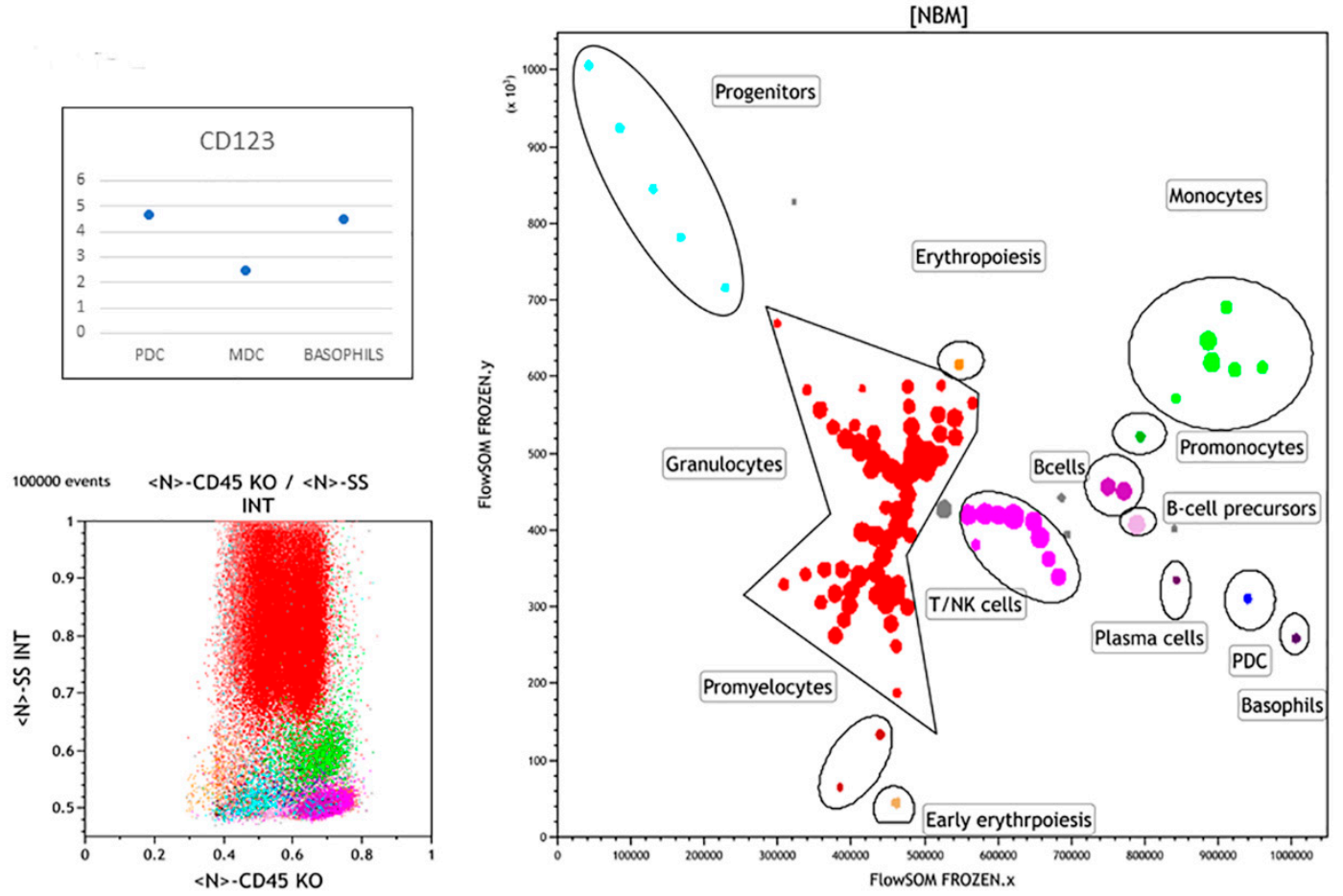

Figure 1. FlowSOM analysis of the merged normal bone marrow files showing the subsets delineated with the applied panel (Supplementary Table S3). Plasmacytoid dendritic cells (PDC) segregate as a single node between basophils and plasma cells on the right side of the minimal spanning tree. The left upper panel shows the normalised MFI expression of CD123 in these subsets and the left lower panel illustrates back-gating of cell subsets on the CD45/SSC plot.

All patient samples were analyzed individually. The FlowSOM set-seed script was run for each sample requesting 100 nodes and six MST proposals. Each of the six MST for each sample was visually checked after back-gating and color-coding of the cell subsets on a standard CD45/SSC histogram. Nodes with marker expression levels and scatter properties similar as those identified in the NBM MST were recognized and color-coded as such. 


\section{Results}

\subsection{Normal Bone Marrow Pattern}

FlowSOM analysis of the merged NBM file (Figure 1) showed 95 nodes that could be identified as cell clusters based on scatter characteristics and CD45 expression. Three nodes showed normalized CD123 expression $>2$. The node characterized as PDC $(0.32 \%$ of all cells, strong CD123 with $4.66 \mathrm{MFI}$ ) was also strongly positive for HLA-DR, CD36 and CD38 with a borderline expression of CD33 (1.52) and intermediate CD45 (0.62). CD7, CD64, CD34 and CD117 were negative (Supplementary Table S3). However, separately performed manual analysis (Supplementary Figure S1) showed in each normal BM a tiny CD7+ population of PDC representing $0.02-0.06 \%$ (mean $0.03 \%$ ) of bone marrow cells and $3-10 \%$ of PDC. Obviously, this population was too small to allow for a separate node by the software. Another node with strong CD123 (MFI 4.47) was characterized as basophils due to negative HLA-DR, stronger CD33 and CD38 expression, all other markers in the panel being negative. The third CD123-positive node was characterized as myeloid DC (MDC) with an intermediate expression of CD123 (MFI 2.5), positivity for HLA-DR, weak CD33 and CD36 but none of the other markers. Marker expressions in other identified cell populations are given in Supplementary Table S3.

\subsection{Abnormal Bone Marrow Samples}

Cases 1-5 (Table 1) were RUNX1 ${ }^{\text {mut }}$ and showed distinct CD123+ subsets, while cases 6-7 had a clear PDC population with some aberrant features but were RUNX1 ${ }^{\text {wt }}$. Cases 1 and 2 (Table 1) were diagnosed as AML with RUNX1 ${ }^{\text {mut }}$. No follow-up samples were available in these cases. Case 3 was classified as AML-MRC due to del(7q) and a sample taken after induction therapy was also be studied. Case 4 was classified as t-AML due to previous chemotherapy for colon cancer. Case 5 was diagnosed as CMML-1, six months before the studied sample was taken. The patient developed a skin lesion with the morphology and immunophenotype of B PDCN. At the time of BPDCN diagnosis there were no signs of BM involvement, and an allogeneic hematopoietic stem cells transplant (AlloHSCT) was planned. The sample taken before Allo-HSCT showed signs of involvement by BPDCN. Another sample taken after Allo-HSCT showed complete remission. Cases 6 and 7 were diagnosed as t-AML and T/myeloid MPAL, respectively. A follow-up sample after induction therapy was studied in case 7 .

\subsubsection{Case 1}

Case 1 is a $R U N X 1^{\text {mut }}$ AML that displayed a dominating $\mathrm{CD} 34^{+} \mathrm{CD} 117^{+} \mathrm{CD} 45^{\mathrm{dim}}$ $\mathrm{CD}^{-}{ }^{-} \mathrm{CD} 33^{-} \mathrm{CD}^{-} 4^{-} \mathrm{CD}^{-}$blast population with low SSC. Detailed analysis by FlowSOM (Figure 2, Supplementary Table S4) showed the near absence of granulocytes and absence of monocytes with five nodes of lymphocytes and four of remaining normal hematopoiesis.

The bulk of the blastic population, quite homogeneous in SSC/CD45, was in fact split into 10 subsets based on differential expression of CD123, HLADR, CD34 and CD38. Three $\mathrm{CD} 34^{+} \mathrm{CD} 117^{+}$subsets showed strong CD123 expression (>3) and differed by HLA-DR and CD38 (from strong through intermediate to dim). Five CD $34^{+} \mathrm{CD} 117^{+}$subsets had intermediate CD123 and varied by the levels of HLA-DR expression. One CD34 $4^{+} \mathrm{CD} 117^{+}$ subset was CD123, CD38 and HLA-DR negative. One subset was CD123 intermediate and CD34 negative but $\mathrm{CD} 117^{+}$. This subset was CD33 positive and had stronger CD45 but was negative for CD36 and CD64, suggesting differentiation towards promyelocytes.

\subsubsection{Case 2}

Case 2 is another $R U N X 1^{\text {mut }}$ AML that had both an immature $\mathrm{CD} 34^{+} \mathrm{CD} 117^{+}$myeloid blast population and a clear population with differentiation towards PDC (Figure 3, Supplementary Table S5). 

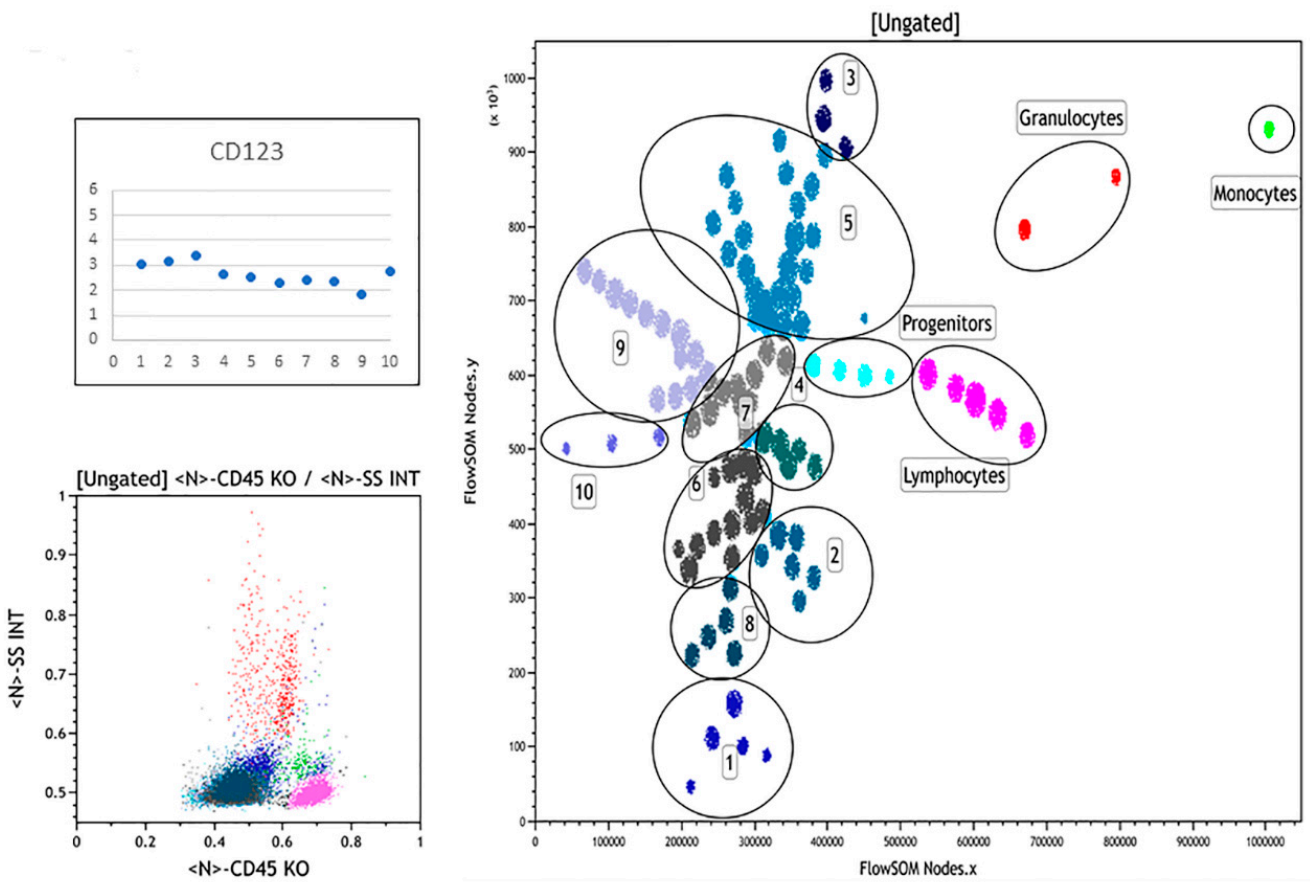

Figure 2. FlowSOM analysis of Case 1 (minimal spanning tree). CD123+ cells constitute a highly clustered population on a classical CD45/SSC scattergram (left lower panel), but FlowSOM (right panel) separates this population in an unsuspectedly large number of nodes that supervised node-by-node analysis cluster in 10 different populations (Supplementary Table S4). The upper left diagram shows the normalised MFI expression of CD123 in numbered cell subsets.
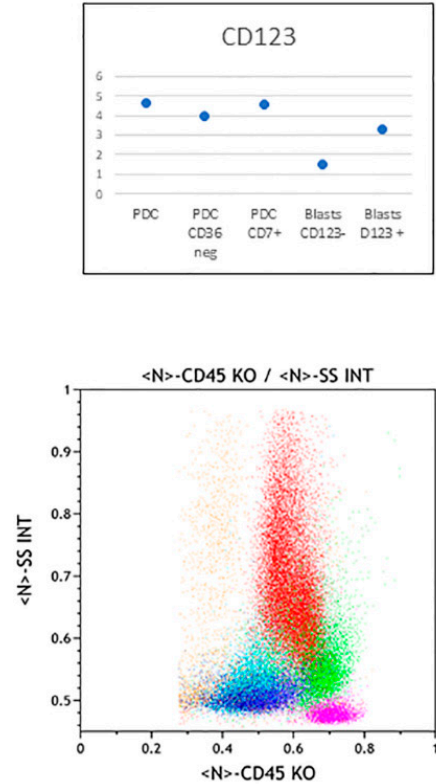

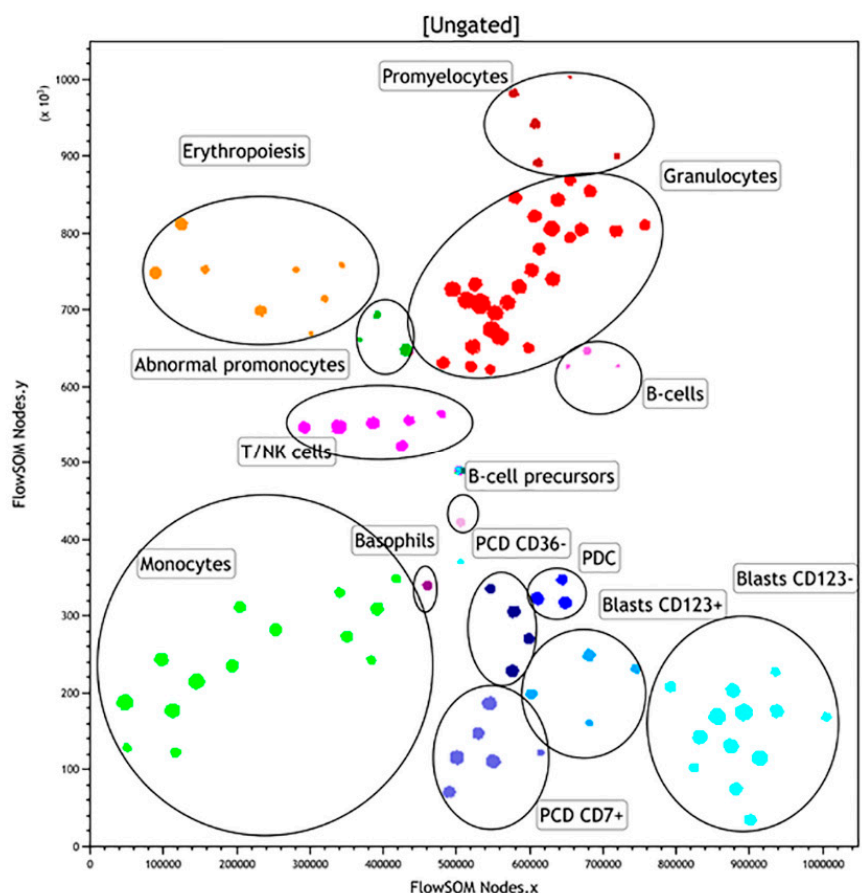

Figure 3. FlowSOM analysis of Case 2. Minimal spanning tree is shown on the right panel. Two different clusters of blast cells are separated on the basis of CD123 expression. Three different PDC clusters are segregated based on CD7 or CD36 expression (Supplementary Table S5). Normalized MFI of CD123 expression in positive cell subsets is given in the upper left diagram and back-gating on the CD45/SSc plot is illustrated on the lower-left panel.

Most blasts were negative for CD123 except for one subset (15\%, Supplementary Table S5). This population had lower CD117 and CD45 expression but higher CD38 and no CD36. The PDCs represented $13.47 \%$ of the cells and were partitioned into three different subsets. 
One had a similar immunophenotype as the PDC node in NBM (Supplementary Table S3) yet with a somewhat lower CD36 expression. The second subset was CD36 negative and the third was CD36 and CD7 positive. Other BM subsets were well represented, with a high diversity among monocytes

\subsubsection{Case 3}

Case 3 was classified as AML-MRC with RUNX1 ${ }^{\text {mut }}$. At diagnosis, $22 \%$ of the cells were identified as blasts with four subsets that differed in CD123, CD33, CD34 and CD117 expression (Supplementary Table S6, Figure 4a).

a
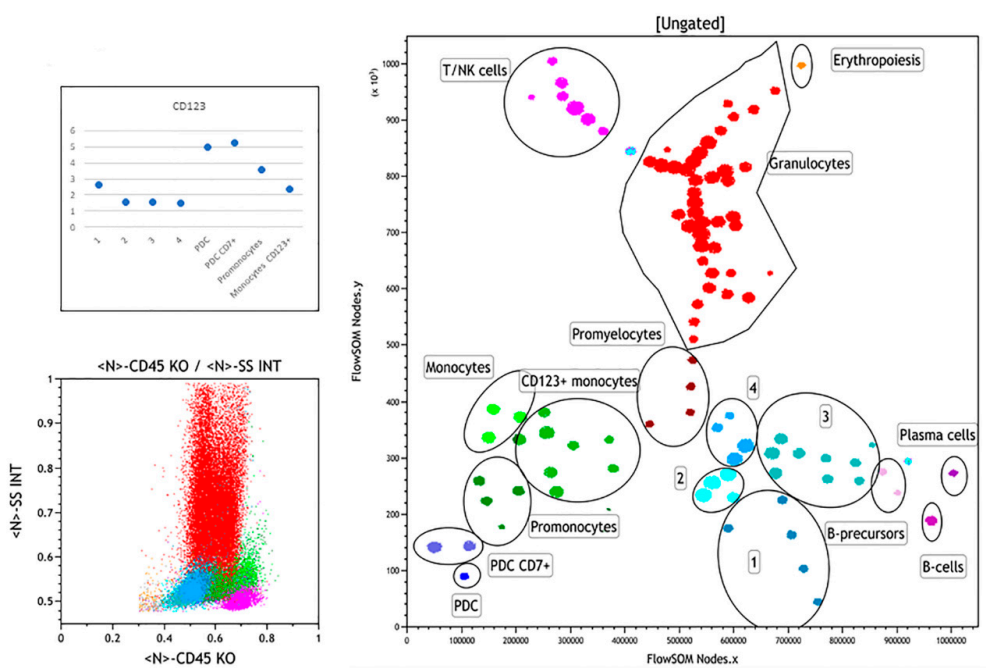

$$
\text { b }
$$
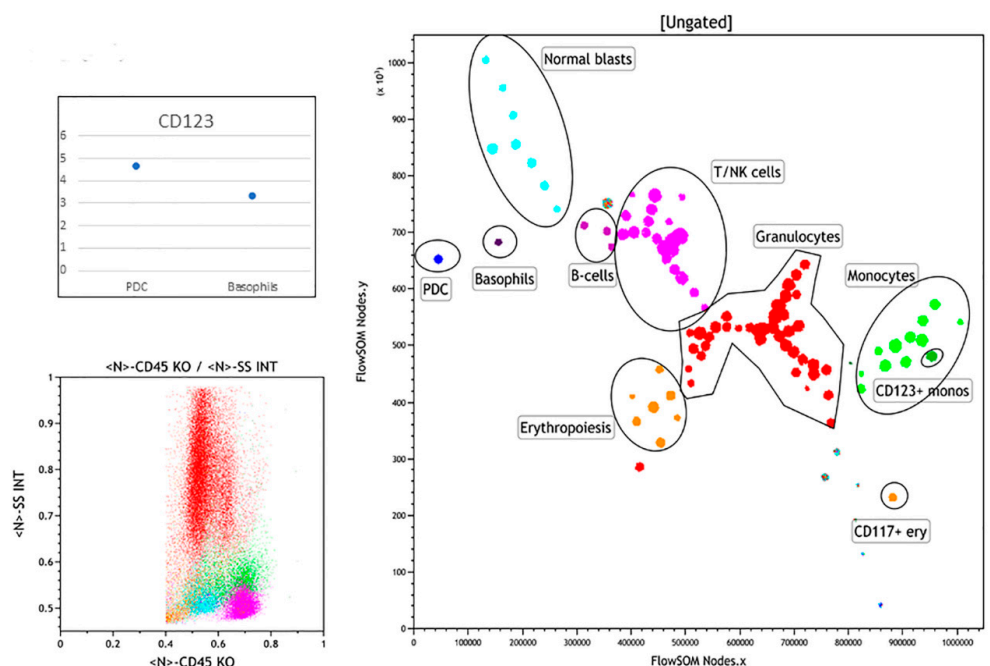

Figure 4. (a) FlowSOM analysis of Case 3 at diagnosis. Minimal spanning tree is given in the right panel. The progenitor population is highly heterogeneous and PDC segregates as two different clusters based on CD7 expression (Supplementary Table S6). Normalised MFI of CD123 expression in numbered progenitor subsets and PDCs is illustrated in the upper left diagram and back-gating on the CD45/SSC plot is given in the lower-left panel. (b) FlowSOM analysis of Case 3 after induction treatment. Minimal spanning tree is shown in the right panel. Normal partition of cell subsets, including recovery of a single classical PDC node is seen. Normalised MFI of CD123 expression in PDCs and basophils is illustrated in the upper-left diagram and back-gating on the CD45/SSC plot is given in the lower-left panel. 
There was a small population of classical PDC and a larger population of $\mathrm{CD}^{+} \mathrm{PDC}$. Moreover, abnormal $\mathrm{CD}_{123^{+}}$populations of promonocytes $\left(\mathrm{CD} 117^{+}\right)$and monocytes were detected (Supplementary Table S6). After induction therapy (Figure 4b, Supplementary Table S6) the BM pattern was almost normal except for a small subset of CD123+ monocytes.

\subsubsection{Case 4}

Case 4 was classified as t-AML with, among other anomalies, RUNX1 ${ }^{\text {mut }}$ (Table 1). FlowSOM analysis (Figure 5, Supplementary Table S7) showed a major ( 74\% of the cells) population of $\mathrm{CD} 34^{+} \mathrm{CD} 117^{+}$blasts with low $\mathrm{CD} 38$ expression and absence of CD123. Amazingly, FlowSOM partitioned this bulk of cells in more than 60 individual nodes. These blasts were $\mathrm{CD} 33$ negative. However, two subsets of $\mathrm{CD} 34^{+} / \mathrm{CD} 117^{+} / \mathrm{CD} 123^{+}$blasts were found: one CD33/CD38 negative and one with CD38 and CD33 expression. Of note a minor population of blasts positive for CD34, CD117 and CD36 was also detected.
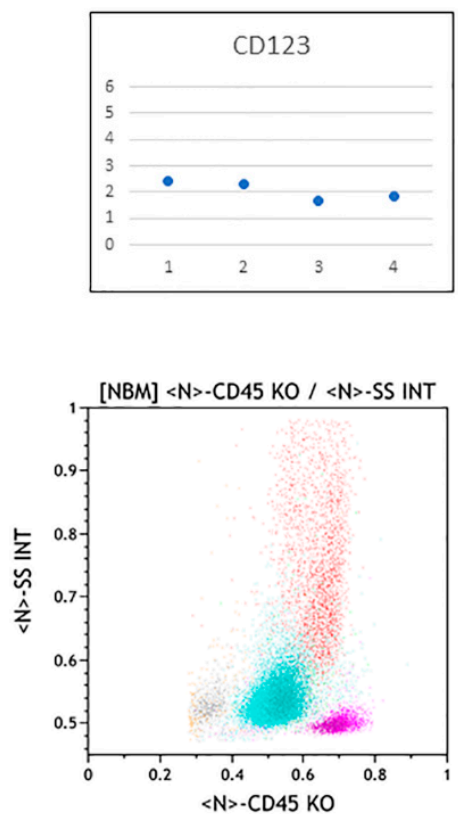

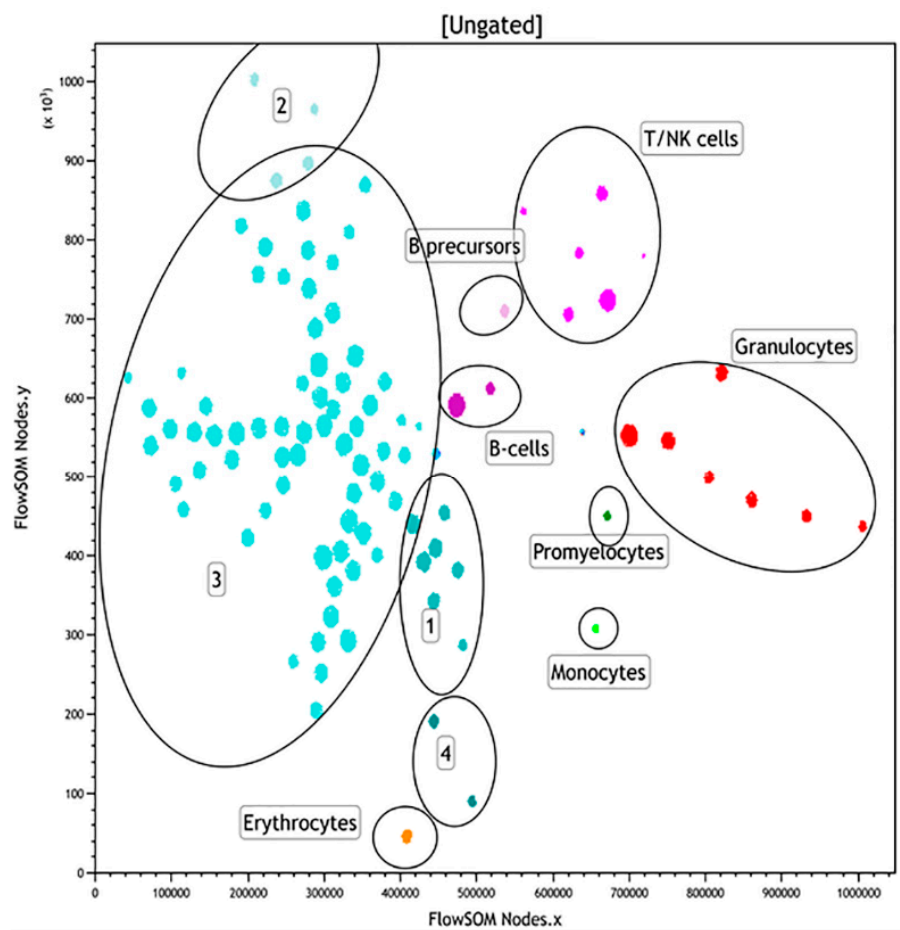

Figure 5. FlowSOM analysis of Case 4. Minimal spanning tree is shown in the right panel. FlowSOM segregates the apparently clustered population of progenitors (left CD45/SSC scattergram) in an impressively large number of nodes that may be re-clustered in 4 populations (Supplementary Table S7). Two subsets of CD34 ${ }^{+} / \mathrm{CD} 117^{+} / \mathrm{CD} 123^{+}$blasts were found: one CD33/CD38 negative and one with CD38 and CD33 expression. Normalised MFI of CD123 expression in numbered progenitor subsets is illustrated in the upper left diagram and back-gating on the CD45/SSC plot is given in the lower-left panel. There is no clear mature PDC population in this case.

\subsubsection{Case 5}

Case 5 is the sample from the CMML patient who was also diagnosed with BPDCN on a skin lesion biopsied 4 months before. At this time, only one normal PDC node was found in the BM sample (data not shown). In the current BM sample collected before Allo-HSCT, routine analysis disclosed $3 \%$ of abnormal $\mathrm{CD} 56^{+} \mathrm{CD} 4^{+} \mathrm{CD} 123^{+} \mathrm{HLA}-\mathrm{DR}{ }^{+}$cells corresponding to early involvement by BPDCN (Figure 6). FlowSOM analysis (Figure 6, Supplementary Table S8) revealed two subsets of CD34+CD117+ blasts $(8.2 \%$ of total) that were CD123 negative and differed by a low CD38 and HLA-DR expression, in one of the subsets. Also, a minor population of $\mathrm{CD} 34^{+} \mathrm{CD} 117^{+}$blasts with $\mathrm{CD} 123$ expression was noted $(0.28 \%)$. Two populations of PDC were observed, one of them coexpressing CD7 (2.8\%) corresponding to the CD56+ population found at routine analysis. Moreover, a diversified population of granulocytes, normal proportions of lymphocytes and four nodes 
of monocytes were found. One month after Allo-HSCT, only $0.78 \%$ of normal PDC were found in the reconstituted BM (data not shown).
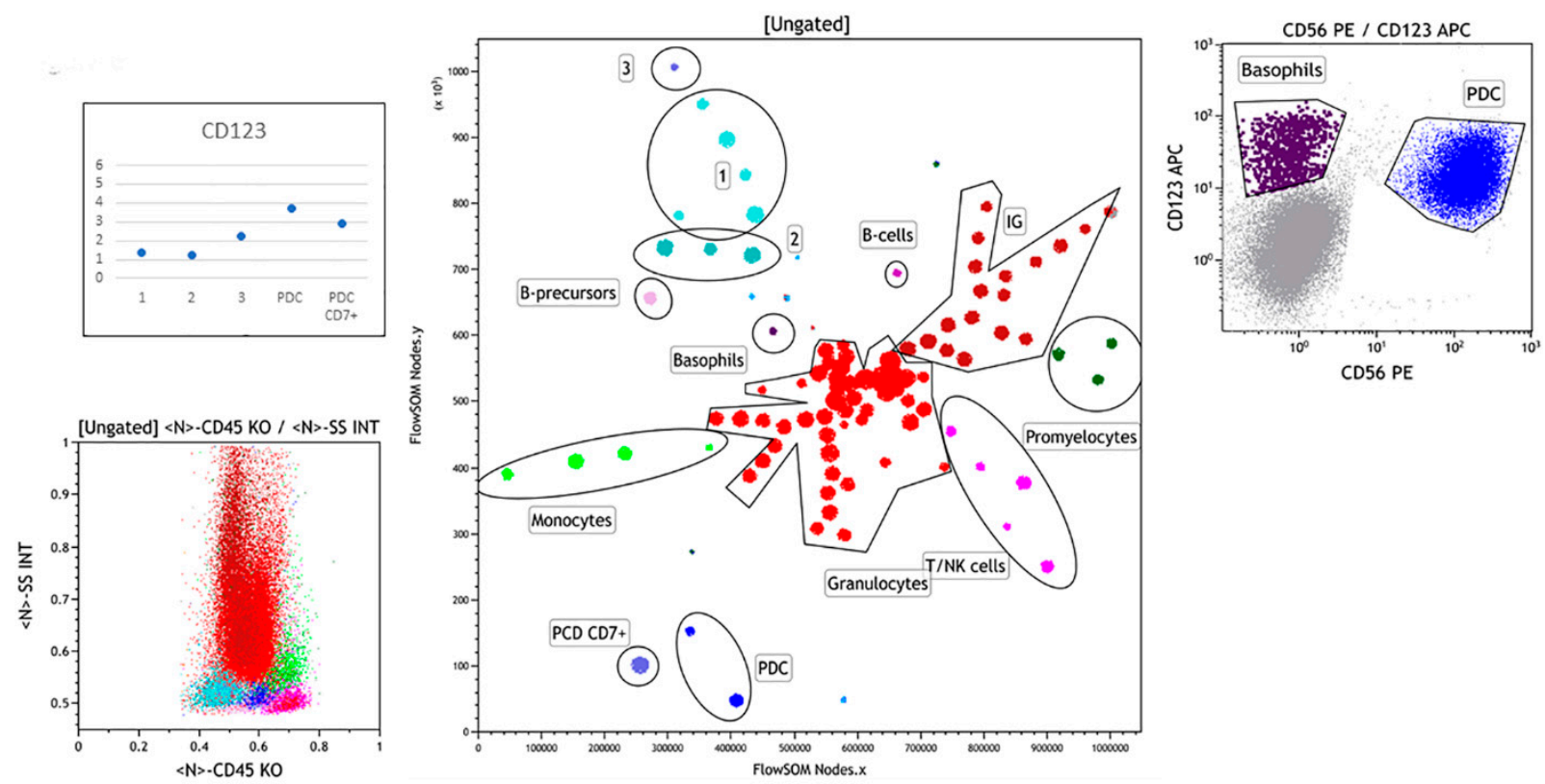

Figure 6. FlowSOM analysis of Case 5. Minimal spanning tree is shown in the middle panel. In this CMML case with evolution towards BPCDN FlowSOM segregates three nodes as normal PDC $(n=2)$ or CD7+ PDC $(n=1)$ that merge in a single cluster in the CD45/SSC scattergram (bottom left) (Supplementary Table S8). Normalised MFI of CD123 expression is given in the upper left diagram. The upper right scattergram, obtained from the same sample analyzed with a BPDCN-focused panel, confirms the CD56+/CD123+ immunophenotype of these cells, clearly separated from the small population of basophils (enhanced display).

\subsubsection{Case 6}

Case 6 was a t-AML and RUNX1 ${ }^{\text {wt }}$ (Table 1). Five CD117+ blast populations could be separated by FlowSOM, including two CD123+ subsets (Figure 7, Supplementary Table S9). Four were CD34+ and two were CD7+. One of the CD123+ subsets had strong CD123 and HLA-DR expression and was CD7 negative, while the other had intermediate CD123 and HLA-DR but was CD7 positive. Moreover, a population of cells identified as PDC precursors strongly expressing CD123 with weak CD34 expression and positive for CD36 and CD7, was noted together with a mature PDC population, also expressing CD7.

\subsubsection{Case 7}

Case 7: Diagnosed as a T/myeloid MPAL due to the presence of populations of blasts coexpressing cyt.CD3, CD7, CD13dim, CD34, CD117, TdT dim, and MPO with low HLADR in a subset of blasts. All other tested T-cell markers (CD1a, CD2, mCD3, CD4, CD8) and CD56 were negative. FlowSOM analysis (Figure 8 and Supplementary Table S10) showed a major population of $\mathrm{CD} 7^{+} \mathrm{CD} 34^{+} \mathrm{CD} 117+\mathrm{CD} 123^{-}$blasts. Another population in the blast region was negative for all markers in the panel except $\mathrm{CD} 38$ and a population showing granulocytic differentiation with CD33 $3^{\mathrm{dim}}$ and CD64 ${ }^{\mathrm{dim}}$ was found. Two populations of CD123 ${ }^{\text {dim }}$ blasts were noted and three subsets of PDC (7.47\% totally) could be delineated (one $\mathrm{CD} 36^{+} \mathrm{CD}^{+}$, one $\mathrm{CD} 36^{+} \mathrm{CD}^{-}$and one negative for both $\mathrm{CD} 36$ and $\mathrm{CD} 7$. 

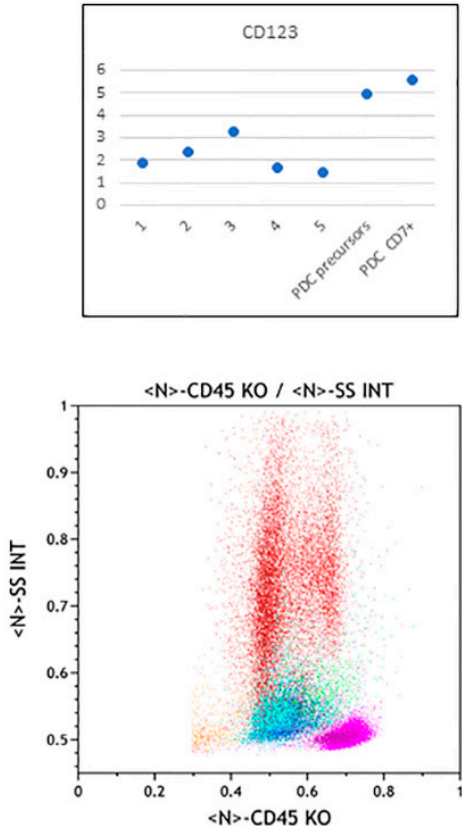

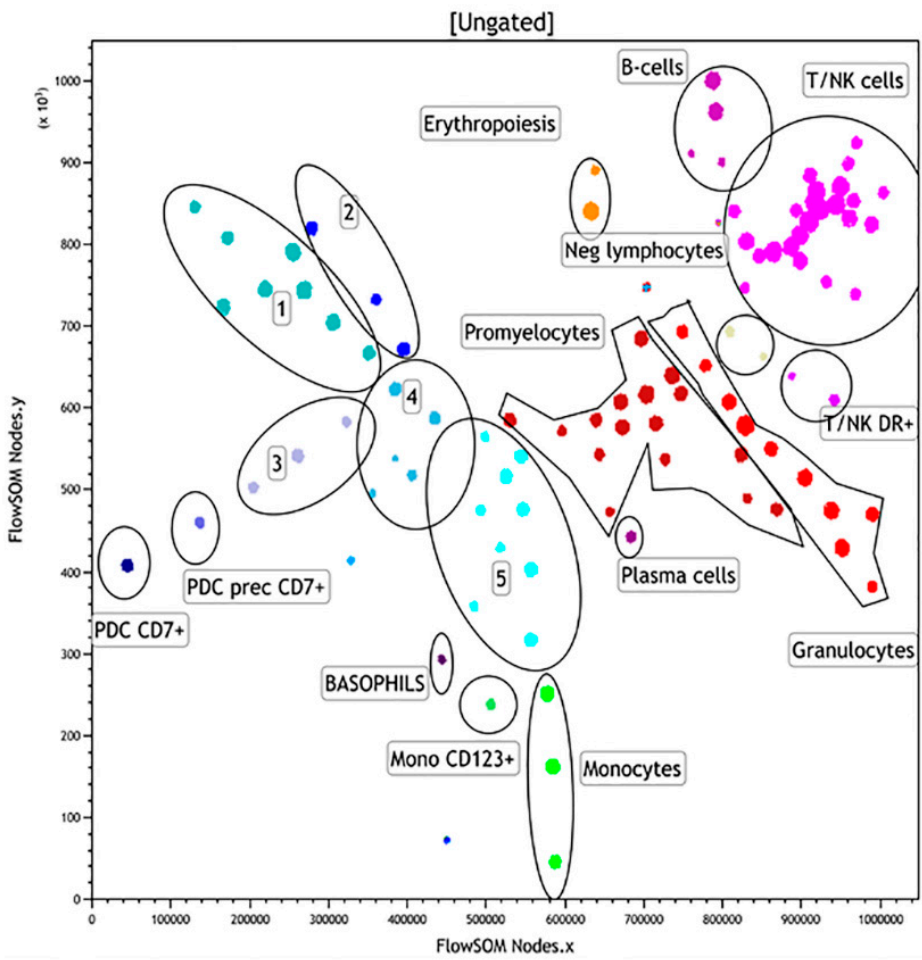

Figure 7. FlowSOM analysis of Case 6. Minimal spanning tree is shown in the right panel. The apparently homogeneous blastic population was objectived by a CD45/SSC scattergram (bottom left) is dismembered in 5 progenitor clusters while PDC segregates in two nodes based on CD34 expression (Supplementary Table S9). Normalised MFI of CD123 expression in numbered subsets of progenitors and PDCs are shown in the upper-left diagram.
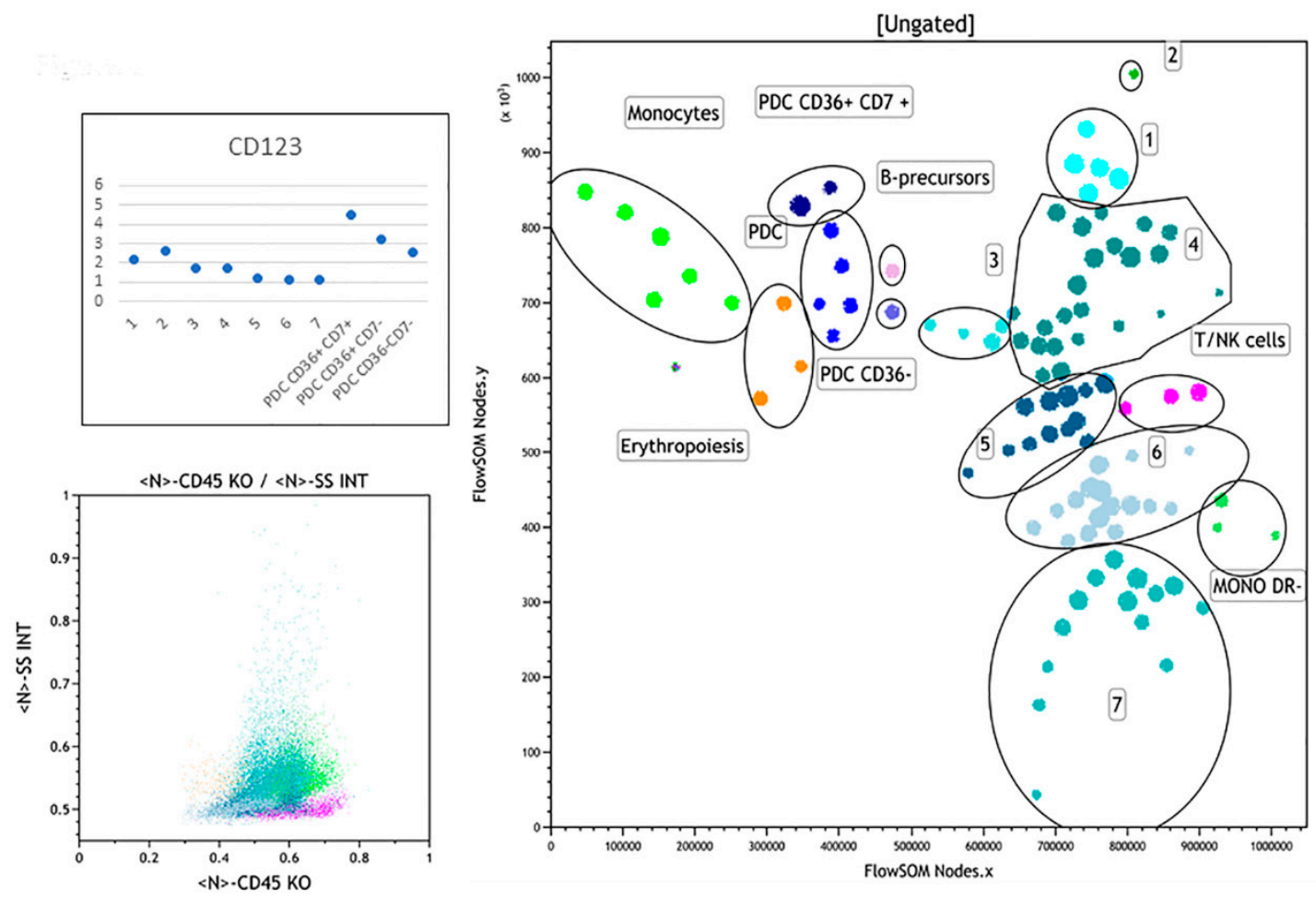

Figure 8. FlowSOM analysis of Case 7. Minimal spanning tree, in the right panel, shows a heavily infiltrated BM as exemplified on the CD45/SSC scattergram (bottom left). The blast population is segregated by FlowSOM in seven progenitor subsets (Supplementary Table S10) and, even more surprisingly, eight PDC subsets (shades of dark blue), four of them being characterized by the absence of CD36 expression and two by the presence of CD7. Normalised MFI of CD123 expression in numbered blast subsets and PDCs is illustrated in the upper left diagram. 


\subsection{Summary of FlowSOM Analysis}

In this series of seven different cases of leukemia, the first striking feature observed after FlowSOM unsupervised analysis is that malignant blastic populations are highly variable from one patient to the other. This underlines the complex interactions that take place in the bone marrow of leukemia patients. Since the algorithm of FlowSOM is designed to segregate the cells in 100 nodes, the diversity of progenitor/blast subsets appeared even greater in cases with almost no remaining normal hematopoiesis (cases \#1, 4 and 7). This novel analysis opens new fields of exploration for a better understanding of leukemogenesis and/or the natural history of the leukemic process.

Only a few common features could be derived from this series where, unexpectedly, RUNX1 mutation was not associated with any specific criterion. Of interest is the observation that "normal" $\mathrm{CD} 36^{+} \mathrm{CD} 7^{-} \mathrm{PDC}$, expressing the highest levels of $\mathrm{CD} 123$ were retrieved in 4/7 samples (Table 2) while CD36 negative PDC subset was found in 2/7 samples. The presence of significant proportions of $\mathrm{CD}^{+} \mathrm{PDC}$ was noted in 5 cases and PDC progenitors were also identified in one case (\# 6).

Table 2. Frequency of $\mathrm{CD}_{123^{+}}$cell subsets in NBM and analyzed patient bone marrows.

\begin{tabular}{|c|c|c|c|c|c|}
\hline Case \# & $\begin{array}{l}\text { Normal PDC } \\
\% \text { Total BM }\end{array}$ & $\begin{array}{c}\text { PDC CD36 } \\
\text { \% Total BM }\end{array}$ & $\begin{array}{l}\text { PDC CD7 }^{+} \\
\% \text { Total BM }\end{array}$ & $\begin{array}{c}\text { MDC } \\
\% \text { Total BM }\end{array}$ & $\begin{array}{l}\text { Basophils } \\
\% \text { Total BM }\end{array}$ \\
\hline NBM & 0.32 & ND & 0.03 & 0.17 & 0.29 \\
\hline 1 & ND & ND & ND & ND & ND \\
\hline 2 & 3.14 & 5.07 & 5.24 & 0.67 & 0.63 \\
\hline 3 & 0.5 & ND & 2.24 & ND & ND \\
\hline 4 & ND & ND & $<0.01$ & $<0.01$ & $<0.01$ \\
\hline 5 & 1.14 & ND & 1.6 & ND & 0.29 \\
\hline 6 & ND & ND & 0.89 & ND & 0.39 \\
\hline 7 & 4.06 & 0.89 & 2.52 & ND & ND \\
\hline
\end{tabular}

\# Patient characteristics and frequency of $\mathrm{CD}_{12} 3^{+}$. blasts/progenitors are given in Table 1; Abbreviations: NBM: normal bone marrow, PDC: plasmacytoid dendritic cells; MDC: myeloid dendritic cells, ND: not detected.

\section{Discussion}

The original approach of FlowSOM-aided unsupervised analysis of FCM data, applied here with a focus on CD123 expression, provides interesting and somehow unexpected new findings. Despite the small size of this series, new information can be obtained from its in-depth analysis. Of note, a high level of variation is derived from the algorithm's set feature of 100 nodes, leading to more heterogeneity observed in samples with heavy blastic infiltration. Yet, node-by-node analysis allows for a secondary supervised review to identify clusters with highly similar features. This could be considered either a limitation of the study or an additional opportunity to examine the plasticity of leukemic blast cells.

More general information can also be derived from this work. The first point of interest is the highly homogenous immunophenotype of normal PDCs, identified as a single node with a typical immunophenotype in a series of merged NBM. This approach also straightforwardly singled out, among $\mathrm{CD} 123^{+}$cells, a unique node of basophils and a unique node of myeloid DC with lower CD123 expression. Since the approach chosen by our group is to work with several samples of merged NBM to obtain the most accurate picture of normal patterns [22,27], our findings indicate that these three CD123+ subsets are robustly defined in NBM.

The stable pattern in normal BM gives even more weight to the astonishing diversity disclosed in the seven cases analyzed here. Indeed, it could have been expected that the RUNX1 ${ }^{\text {mut }}$ signature would be associated with PDC differentiation $[6,7]$ but several intriguing facts may be noted.

The first one is the observation in 4/7 cases of subsets of mature PDC with the expression of CD7. The significance of this population and its possible normal counterpart are not clearly defined. A subset of $\mathrm{CD}^{+}$PDCs in non-neoplastic BM samples was 
described by Wang et al. [18] with a median frequency of $13 \%$ of PDCs (range, $0.3 \%$ to $21 \%$ ). This population was not singled out by the present FlowSOM analysis of NBM, although it could be retrieved by supervised manual analysis. The presence of $\mathrm{CD} 7^{+} \mathrm{PDC}$ certainly fits the earliest definition of PDC as lymphoid-derived (or, more precisely, T-lymphoid). This concept has again gained interest, and a pathway for the PDC lineage to develop from a lymphoid progenitor that runs parallel to B-cell development rather than being linked to the myeloid branch of common dendritic cell precursors was proposed [29]. Populations of PDCs detected by FlowSOM in neoplastic cases studied here show variability and, in some cases, a small subset suggesting the presence of PDC precursors was found. This was not fully described in previous studies [30].

Another interesting feature is that of the immunophenotypic variability of blast subsets associating with CD123 expression, suggesting perhaps their dependency on IL-3. If this is further demonstrated, the use of CD123 targeted therapies such as tagraxofusp, CD123directed CAR T-cells, or bi-specifics could be better documented [31]. Xiao et al. [7] demonstrated in xenograft models of PDC-AML that targeting with tagraxofusp led to the elimination of malignant PDCs and a two- to three-fold decrease in leukemia tumor burden, but not full elimination of leukemia.

A third important point, also regarding the potential therapeutic issues just mentioned, is that FlowSOM sequential analysis of patients' BM can provide a more precise appreciation of treatment efficacy. It is of major interest that in the three follow-up samples that were obtained in this series, normal PDCs and CD123 patterns were completely recovered after treatment.

In summary, although an extensive dissection of the subsets identified here is beyond the scope of this preliminary report, the presented analyses certainly point out the extraordinary flexibility of malignant cells in their immunophenotypic marker expression. Unsupervised analysis, although somewhat "simplified" here by grouping node clusters that are considered highly similar, discloses both the impressive homeostasis of NBM and the abundance of variegating patterns displayed by malignant cells. The relationship with molecular anomalies, which are just as variable, remains to be deciphered.

Supplementary Materials: The following are available online at https: / www.mdpi.com/article / 10.3390/hemato2030036/s1: Supplementary Methods, Supplementary Figure S1 A minimal CD7+ population of PDC detected in normal bone marrow (one of the samples of merged NBM file), Table S1: Combinations of antibodies in the comprehensive myeloid panel (AML Tubes 1-3) applied at diagnosis. Table S2: Cut-offs for the classification of normalized marker expressions in FlowSOM analysis of AML tube 2. Supplementary Table S3: Cell subsets identified by FlowSOM in the merged file of six normal BM analyzed with panel AML 2 and their respective normalized MFI. Supplementary Table S4: Cell subsets identified by FlowSOM in Case 1 and their respective normalized MFI. Supplementary Table S5: Cell subsets identified by FlowSOM in Case 2 and their respective normalized MFI. Supplementary Table S6: Cell subsets identified by FlowSOM in Case 3 and their respective normalized MFI. Supplementary Table S7: Cell subsets identified by FlowSOM in Case 4 and their respective normalized MFI. Supplementary Table S8: Cell subsets identified by FlowSOM in Case 5 and their respective normalized MFI. Supplementary Table S9: Cell subsets identified by FlowSOM in Case 6 and their respective normalized MFI. Supplementary Table S10: Cell subsets identified by FlowSOM in Case 7 and their respective normalized MFI.

Author Contributions: Both authors contributed equally to this work. Both authors have read and agreed to the published version of the manuscript.

Funding: This research received no external funding.

Institutional Review Board Statement: The list-mode files analyzed in this in silico study were those of routine flow-cytometry analysis. All information was taken from pathology reports. The patient charts were not reviewed.

Informed Consent Statement: Patient consent was waived due to de-identified data. Normal donor samples were obtained with the approval of the local ethics committee. 
Data Availability Statement: FCS files couls be made available upon request.

Acknowledgments: The cases were diagnosed at the Department of Pathology and flow cytometry was run at the Flow Cytometry Laboratory Lund University Hospital, Lund, Sweden. Routine cytogenetic and molecular analyses were performed at the Department of Cytogenetics, Lund University Hospital, Lund, Sweden.

Conflicts of Interest: The authors declare no conflict of interest.

\section{References}

1. Sood, R.; Kamikubo, Y.; Liu, P. Role of RUNX1 in hematological malignancies. Blood 2017, 129, 2070-2082. [CrossRef]

2. Gaidzik, V.I.; Teleanu, V.; Papaemmanuil, E.; Weber, D.; Paschka, P.; Hahn, J.; Wallrabenstein, T.; Kolbinger, B.; Köhne, C.H.; Horst, H.A.; et al. RUNX1 mutations in acute myeloid leukemia are associated with distinct clinico-pathologic and genetic features. Leukemia 2016, 11, 2160-2168. [CrossRef] [PubMed]

3. Swerdlow, S.; Campo, E.; Harris, N.; Jaffz, E.S.; Pileri, S.A.; Stein, H.; Thiele, J. (Eds.) WHO Classification of Tumours of Haematopoietic and Lymphoid Tissues, 4th ed.; IARC: Lyon, France, 2017.

4. George, T.I.; Bajel, A. Diagnosis of rare subtypes of acute myeloid leukaemia and related neoplasms. Pathology 2021, 3, 312-327. [CrossRef]

5. Takahashi, K.; Wang, F.; Morita, K.; Yan, Y.; Hu, P.; Zhao, P.; Zhar, A.A.; Wu, C.J.; Gumbs, C.; Little, L.; et al. Integrative genomic analysis of adult mixed phenotype acute leukemia delineates lineage associated molecular subtypes. Nat. Commun. 2018, 1, 2670. [CrossRef]

6. Zalmaï, L.; Viailly, P.J.; Biichle, S.; Cheok, M.; Soret, L.; Angelot-Delettre, F.; Petrella, T.; Collonge-Rame, M.A.; Seilles, E.; Geffroy, S.; et al. Plasmacytoid dendritic cells proliferation associated with acute myeloid leukemia: Phenotype profile and mutation landscape. Haematologica 2020. [CrossRef] [PubMed]

7. Xiao, W.; Chan, A.; Waarts, M.R.; Mishra, T.; Liu, Y.; Cai, S.F.; Yao, J.; Gao, Q.; Bowman, R.L.; Koche, R.P.; et al. Plasmacytoid dendritic cell expansion defines a distinct subset of RUNX1-mutated acute myeloid leukemia. Blood 2021, 137, $1377-1391$. [CrossRef]

8. Renosi, F.; Roggy, A.; Giguelay, A.; Soret, L.; Viailly, P.J.; Cheok, M.; Biichle, S.; Angelot-Delettre, F.; Asnafi, V.; Macintyre, E.; et al. Transcriptomic and genomic heterogeneity in blastic plasmacytoid dendritic cell neoplasms: From ontogeny to oncogenesis. Blood Adv. 2021, 5, 1540-1551. [CrossRef]

9. Naresh, K.N.; Pavlu, J. Plasmacytoid dendritic cell nodules in bone marrow biopsies of chronic myelomonocytic leukemia. Am. J. Hematol. 2010, 85, 89. [CrossRef] [PubMed]

10. Vitte, F.; Fabiani, B.; Bénet, C.; Dalac, S.; Balme, B.; Delattre, C.; Vergier, B.; Beylot-Barry, M.; Vignon-Pennamen, D.; Ortonne, N.; et al. Specific skin lesions in chronic myelomonocytic leukemia: A spectrum of myelomonocytic and dendritic cell proliferations: A study of 42 cases. Am. J. Surg. Pathol. 2012, 36, 1302-1316. [CrossRef]

11. Brunetti, L.; Di Battista, V.; Venanzi, A.; Schiavoni, G.; Martelli, M.P.; Ascani, S.; Mecucci, C.; Tiacci, E.; Falini, B. Blastic plasmacytoid dendritic cell neoplasm and chronic myelomonocytic leukemia: A shared clonal origin. Leukemia 2017, 31, 12381240. [CrossRef]

12. Adnan Awad, S.; Dufva, O.; Ianevski, A.; Ghimire, B.; Koski, J.; Maliniemi, P.; Thomson, D.; Schreiber, A.; Heckman, C.A.; Koskenvesa, P.; et al. RUNX1 mutations in blast-phase chronic myeloid leukemia associate with distinct phenotypes, transcriptional profiles, and drug responses. Leukemia 2021, 35, 1087-1099. [CrossRef] [PubMed]

13. Facchetti, F.; de Wolf-Peeters, C.; Mason, D.Y.; Pulford, K.; van den Oord, J.J.; Desmet, V.J. Plasmacytoid T cells. Immunohistochemical evidence for their monocyte/macrophage origin. Am. J. Pathol. 1988, 133, 15-21.

14. Chaperot, L.; Bendriss, N.; Manches, O.; Gressin, R.; Maynadie, M.; Trimoreau, F.; Orfeuvre, H.; Corront, B.; Feuillard, J.; Sotto, J.J.; et al. Identification of a leukemic counterpart of the plasmacytoid dendritic cells. Blood 2001, 97, 3210-3217. [CrossRef] [PubMed]

15. Galibert, L.; Maliszewski, C.R.; Vandenabeele, S. Plasmacytoid monocytes/T cells: A dendritic cell lineage? Semin. Immunol. 2001, 13, 283-289. [CrossRef] [PubMed]

16. Feuillard, J.; Jacob, M.C.; Valensi, F.; Maynadié, M.; Gressin, R.; Chaperot, L.; Arnoulet, C.; Brignole-Baudouin, F.; Drénou, B.; Duchayne, E.; et al. Clinical and biologic features of CD4(+)CD56(+) malignancies. Blood 2002, 99, 1556-1563. [CrossRef] [PubMed]

17. Garnache-Ottou, F.; Feuillard, J.; Ferrand, C.; Biichle, S.; Trimoreau, F.; Seilles, E.; Salaun, V.; Garand, R.; Lepelley, P.; Maynadié, M.; et al. Extended diagnostic criteria for plasmacytoid dendritic cell leukaemia. Br. J. Haematol. 2009, 145, 624-636. [CrossRef]

18. Wang, W.; Khoury, J.D.; Miranda, R.N.; Jorgensen, J.L.; Xu, J.; Loghavi, S.; Li, S.; Pemmaraju, N.; Nguyen, T.; Medeiros, L.J.; et al. Immunophenotypic characterization of reactive and neoplastic plasmacytoid dendritic cells permits establishment of a 10-color flow cytometric panel for initial workup and residual disease evaluation of blastic plasmacytoid dendritic cell neoplasm. Haematologica 2021, 106, 1047-1055. [CrossRef]

19. Breton, G.; Lee, J.; Liu, K.; Nussenzweig, M.C. Defining human dendritic cell progenitors by multiparametric flow cytometry. Nat. Protoc. 2015, 10, 1407-1422. [CrossRef]

20. Musumeci, A.; Lutz, K.; Winheim, E.; Krug, A.B. What Makes a PDC: Recent advances in understanding plasmacytoid dc development and heterogeneity. Front. Immunol. 2019, 10, 1222. [CrossRef] 
21. Deotare, U.; Yee, K.W.; Le, L.W.; Porwi, T.A.; Tierens, A.; Musani, R.; Barth, D.; Torlakovic, E.; Schimmer, A.; Schuh, A.C.; et al. Blastic plasmacytoid dendritic cell neoplasm with leukemic presentation: 10-Color flow cytometry diagnosis and HyperCVAD therapy. Am. J. Hematol. 2016, 91, 283-286. [CrossRef]

22. Lacombe, F.; Dupont, B.; Lechevalier, N.; Vial, J.P.; Béné, M.C. Innovation in flow cytometry analysis: A new paradigm delineating normal or diseased bone marrow subsets through machine learning. Hemasphere 2019, 3, e173. [CrossRef]

23. Cheung, M.; Campbell, J.J.; Whitby, L.; Thomas, R.J.; Braybrook, J.; Petzing, J. Current trends in flow cytometry automated data analysis software. Cytom. A 2021. [CrossRef]

24. Van Gassen, S.; Callebaut, B.; Van Helden, M.J.; Lambrecht, B.N.; Demeester, P.; Dhaene, T.; Saeys, Y. FlowSOM: Using selforganizing maps for visualization and interpretation of cytometry data. Cytom. A 2015, 87, 636-645. [CrossRef] [PubMed]

25. Lacombe, F.; Lechevalier, N.; Vial, J.P.; Béné, M.C. An R-derived flowsom process to analyze unsupervised clustering of normal and malignant human bone marrow classical flow cytometry data. Cytom. A 2019, 95, 1191-1197. [CrossRef] [PubMed]

26. Vial, J.P.; Lechevalier, N.; Lacombe, F.; Dumas, P.Y.; Bidet, A.; Leguay, T.; Vergez, F.; Pigneux, A.; Béné, M.C. Unsupervised flow cytometry analysis allows for an accurate identification of minimal residual disease assessment in acute myeloid leukemia. Cancers 2021, 13, 629. [CrossRef] [PubMed]

27. Béné, M.C.; Axler, O.; Violidaki, D.; Lacombe, F.; Ehinger, M.; Porwit, A. Definition of erythroid differentiation subsets in normal human bone marrow using FlowSOM unsupervised cluster analysis of flow cytometry data. Hemasphere 2020, 5, e512. [CrossRef]

28. Violidaki, D.; Axler, O.; Jafari, K.; Bild, F.; Nilsson, L.; Mazur, J.; Ehinger, M.; Porwit, A. Analysis of erythroid maturation in the nonlysed bone marrow with help of radar plots facilitates detection of flow cytometric aberrations in myelodysplastic syndromes. Cytom. B Clin. Cytom. 2020, 98, 399-411. [CrossRef]

29. Rodrigues, P.F.; Tussiwand, R. Novel concepts in plasmacytoid dendritic cell (PDC) development and differentiation. Mol. Immunol. 2020, 126, 25-30. [CrossRef]

30. Hamadeh, F.; Awadallah, A.; Meyerson, H.J.; Beck, R.C. Flow cytometry identifies a spectrum of maturation in myeloid neoplasms having plasmacytoid dendritic cell differentiation. Cytom. B Clin. Cytom. 2020, 98, 43-51. [CrossRef] [PubMed]

31. Sapienza, M.R.; Pileri, A.; Derenzini, E.; Melle, F.; Motta, G.; Fiori, S.; Calleri, A.; Pimpinelli, N.; Tabanelli, V.; Pileri, S. Blastic plasmacytoid dendritic cell neoplasm: State of the art and prospects. Cancers 2019, 11, 595. [CrossRef] 